\title{
Influence of Continuous Bending Process on Texture Evolution and Mechanical Properties of AZ31 Magnesium Alloy
}

\author{
Ting-Zhuang Han ${ }^{1,2}$ • Guang-Sheng Huang ${ }^{1,2,3} \cdot$ Lun Huang $^{1,2} \cdot$ Bin Jiang $^{1,2,3} \cdot$ Guan-Gang Wang ${ }^{1,2}$ • \\ Ai-Tao Tang ${ }^{1,2,3} \cdot$ Fu-Sheng Pan ${ }^{1,2,3}$
}

Received: 24 June 2017/Revised: 18 October 2017/Published online: 15 November 2017

(C) The Chinese Society for Metals and Springer-Verlag GmbH Germany 2017

\begin{abstract}
Mg}-3 \mathrm{Al}-1 \mathrm{Zn}$ alloy sheets have been deformed by continuous bending (CB) to investigate the effects of processing parameters (bending angle and repetitive passes) on the texture and formability. The samples exhibited a bimodal microstructure with abnormal growth grains in the surface region and fine grains distributed in the center after CB process followed by annealing. The texture evolution measured by XRD indicated that the basal poles were rotated from ND toward RD, and the texture intensity decreased with the bending angle decreasing and repetitive passes increasing. Compared with the as-received sample, the yield strength of CBA-120-2 sample significantly decreased from 183 to $112 \mathrm{MPa}$, and a smaller $r$-value and a larger $n$-value were obtained. The formability of CB processed samples in annealing condition was significantly enhanced with the highest of Erichsen value of $5.4 \mathrm{~mm}$, increased by about $135 \%$. The improvement of formability was likely attributed to the weakened and RD-tilt basal texture and coarse grains in the surface part.
\end{abstract}

KEY WORDS: Magnesium alloy; Continuous bending; Microstructure; Texture; Stretch formability

\section{Introduction}

As the lightest metal structural materials, magnesium alloys are of great interest in the 3C, transportation and aerospace industries due to their low density, high specific strength, high specific stiffness, recyclability and so on [1-3]. However, magnesium alloy sheets exhibit poor

Available online at http://link.springer.com/journal/40195

Guang-Sheng Huang

gshuang@cqu.edu.cn

1 State Key Laboratory of Mechanical Transmission, College of Materials Science and Engineering, Chongqing University, Chongqing 400044, China

2 National Engineering Research Center for Magnesium Alloys, Chongqing University, Chongqing 400044, China

3 Chongqing Research Center for Advanced Materials, Chongqing Academy of Science \& Technology, Chongqing 401123, China ductility and formability at ambient temperature due to their hexagonal close-packed (HCP) crystal structure, which hinder their widespread industrial applications. In magnesium alloys, the critical resolved shear stresses (CRSS) for basal slip are much lower than those of the nonbasal slips such as prismatic and pyramidal slips. Hence, plastic deformation of magnesium alloy sheets has been thought to occur almost entirely on the basal planes. However, basal planes can provide only two independent slip systems which cannot satisfy the Von-Mises requirement five independent slip systems for homogeneous deformation $[4,5]$.

In addition, the hot-rolled (or extruded) magnesium alloy sheets usually exhibit a strong basal texture, with the (0002) basal planes distributed parallel to the RD-TD (ED-TD) plane, where the RD, ED and TD represent the rolling, extrusion and transverse direction, respectively. In such a case, it is very difficult for textured sheets to be further deformed along thickness direction, resulting in an obvious anisotropy with a poor stretch formability [6]. 
Therefore, it is important to modify the basal texture by the secondary processing for the purpose of enhancing the formability of magnesium alloy sheets [7, 8].

To date, numerous efforts have been devoted to improve the room temperature formability of magnesium alloy sheets by modifying basal texture. The methods of differential speed rolling (DSR) [9, 10], cross-roll rolling (CR) $[11,12]$ and equal-channel angular pressing (ECAP) $[13,14]$ were proved to be effective to tailor the texture of magnesium alloy sheets. The magnesium alloy sheets underwent these processes exhibited a weakened and random texture, which lead to a significant improvement of formability. In addition, our previous studies [15, 16] revealed that repeated unidirectional bending (RUB) process could effectively modify the basal texture of magnesium alloy sheets and improve the formability. However, the approaches mentioned above have a common limitation as they need to be conducted multi-passes deformation to control the texture and require long process time. Thus, it is difficult to increase the process efficiency, posing a hurdle to the industrial mass processing of magnesium alloys.

For this reason, our previous studies [17, 18] put forward a time-efficient method of continuous bending (CB) process, which introduced multiple times bending in a single pass. It was carried out on an AZ31 magnesium alloy and could improve the formability of magnesium alloy sheets by weakening the basal texture of sheets. However, the parameters (bending angle and repetitive passes) were out of consideration. Thus, the current work aims to investigate the effects of $\mathrm{CB}$ process parameters on the mechanical properties and basal texture of AZ31 magnesium alloy.

\section{Experimental Procedure}

The material used in this work is a commercial AZ31 $(\mathrm{Mg}-$ $3 \% \mathrm{Al}-1 \% \mathrm{Zn}$ ) magnesium alloy sheets with $0.6 \mathrm{~mm}$ thickness. For continuous bending process, the sheets with a dimension of $1000 \mathrm{~mm} \quad(\mathrm{RD}) \times 100 \mathrm{~mm} \quad$ (TD) $\times$ $0.6 \mathrm{~mm}(\mathrm{ND})$ were machined. The RD, TD and ND represent the rolling direction, transverse direction and normal direction, respectively. Then, the machined sheets were continuously bent along RD with constant bending speed of $2.9 \mathrm{~m} / \mathrm{min}$. The schematic diagram of continuous bending device is shown in Fig. 1. The bending angles $\theta$ are $140^{\circ}$ and $120^{\circ}$, and totally two passes were conducted. All the sheets underwent by $\mathrm{CB}$ process were annealed at $260{ }^{\circ} \mathrm{C}$ for $1 \mathrm{~h}$ to acquire a fully recrystallized microstructure. The continuous bent samples and subsequently annealed samples were marked as CB and CBA samples, respectively, such as CB-120-1 and CBA-120-1. 120 is the bending angle and 1 represents one pass.

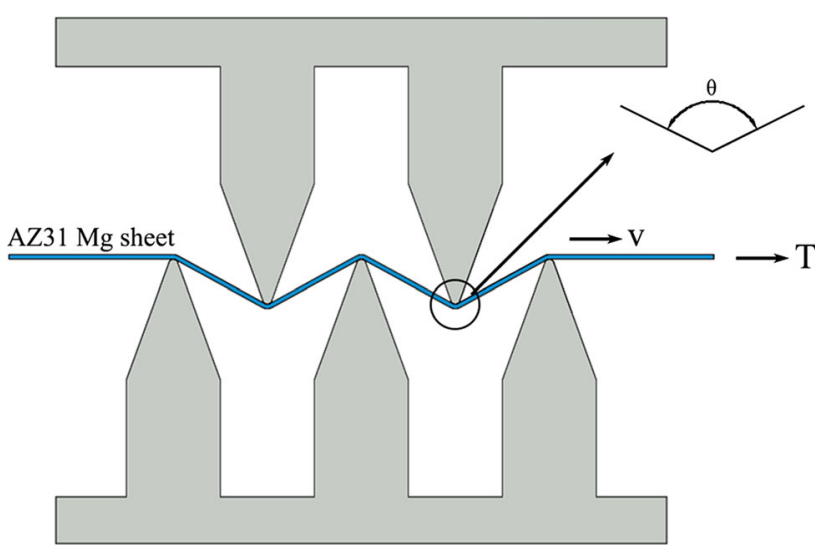

Fig. 1 Schematic diagram of a continuous bending device

Microstructural characteristics were carried out by optical microscopy (OM). The average grain size of all samples was measured by the linear intercept method. The (0002) pole figures of different samples were determined using an X-ray diffraction (XRD, Rigaku D/Max 2500PC) with $\mathrm{Cu} K_{\alpha}$ radiation, a voltage of $40 \mathrm{kV}$ and a current of $100 \mathrm{~mA}$. Fracture morphologies on the surfaces of tensile samples were observed in detail by scanning electron microscopy (SEM, TESCAN VEGA 3 LMH SEM).

Tensile samples were prepared with a gage length of $40 \mathrm{~mm}$ and width of $8 \mathrm{~mm}$. Uniaxial tensile tests were performed on a CMT6305-300KN electronic universal testing machine along three directions, (RD, TD and $45^{\circ}$ ). Minimum of three tests for various samples and directions were conducted, and the data presented is mean of three tests. The mechanical properties namely yield strength (YS), ultimate tensile strength (UTS) and fracture elongation (FE) were obtained from the uniaxial tensile tests. The $n$-values were got from the uniform plastic deformation region of the tensile stress-strain curves. Lankford values ( $r$-values) were the ratio of width strain and thickness strain, which were obtained from the tensile samples deformed to a tensile direction strain of $10 \%$.

The stretch formability was evaluated by Erichsen tests at room temperature. Rectangular blanks with dimension of $50 \mathrm{~mm} \times 50 \mathrm{~mm}$ (width $\times$ length) were cut from the asreceived and CBA samples. The Erichsen tests were carried out using a hemispherical punch with a diameter of $20 \mathrm{~mm}$. The punch speed and the blank holder force were set at $3 \mathrm{~mm} / \mathrm{min}$ and $10 \mathrm{kN}$, respectively.

\section{Results and Discussion}

\subsection{Microstructure Evolution After CB Process}

Figure 2 shows the optical microstructure and (0002) pole figure of the as-received (AS) sample. As shown in Fig. 2a, 
the microstructure consists of equiaxed grains without twins in the AS sample, and the average grain size is $5.8 \mu \mathrm{m}$. Figure $1 \mathrm{~b}$ indicates that the AS sample exhibits a typical rolling texture with the c-axes of most grains aligned parallel to normal direction (ND) and the maximum intensity is 9.5 .

The optical microstructures of the CB-120-1 sample at different regions are shown in Fig. 3. In the I region, the sheet was bent for one time, and the sheet in II region was bent for twice, and the sheet in the III region meant the sheet was completed CB process. The concave of the sheet in I region is defined as upper surface and the convex is defined as lower surface. It is interesting to note that the microstructures did not change after different times bending processing at center and surface part. The deformed microstructural observation of the fine-grained samples with average grain size of $5.5 \mu \mathrm{m}$ shows no existence of twins through the thickness in different regions. During $\mathrm{V}$-bending, grains in convex region are under tensile stress, while the grains in concave region are in compressive stress. Previous studies $[19,20]$ reported that prismatic slip dominated the deformation in convex region, and $\{10-12\}$ extension twins dominated the deformation in concave region. The absence of $\{10-12\}$ extension twins in this work may be owing to the fine-grain microstructure. It is well known that twinning has a great sensitivity to grain size. The activity of $\{10-12\}$ extension twinning can be suppressed by grain refinement and no evident twinning occurred in smaller-sized grains [21, 22]. For the condition of absence of twins, slip modes need to be active for deformation. The Schmid factor for the basal slip of grains in the concave region with the c-axes parallel to the applied loading direction is almost zero; the activation of non-basal slip is required to coordinate the deformation. Previous studies reported that the plastic strain was most likely accommodated by the pyramidal $<\mathrm{c}+\mathrm{a}>$ slip during compression [22-25]. In this work, the grains in the concave region were under compression and the pyramidal $<\mathrm{c}+\mathrm{a}>$ slip may be the preferred deformation mode. Therefore, the prismatic slip may dominate the convex region and the pyramidal $<\mathrm{c}+\mathrm{a}>$ slip dominate concave region for a finegained magnesium alloy in this work.

\subsection{Texture Evolution After CB Process in Annealing Condition}

The evolution of the microstructures after annealing of the CBA-140-1, CBA-120-1 and CBA-120-2 samples is illustrated in Fig. 4. After annealing, static recrystallization occurred through the whole thickness and abnormal grain growth occurred in the surface part of all the annealed samples. This results in characteristics of bimodal microstructure, with small equiaxed grains in the center part and abnormal growth grains in the surface part. For all the CBA samples, the grains in the center part changed a little with the size of $6.0 \mu \mathrm{m}$, while grains in the surface part have a minimum size of $9 \mu \mathrm{m}$ and maximum of $62 \mu \mathrm{m}$. The depth $h$ of abnormal growth grains for the CBA-140-1, CBA-120-1 and CBA-120-2 samples are about 70,115 and $150 \mu \mathrm{m}$, respectively. The abnormal grain growth may be attributed to the heterogeneity deformation through the whole thickness of sheets. The amount of deformation of the surface part was higher than that in the center part, resulting that grains near the surface grew obviously and there was no significant difference in the center part during annealing [26]. This trend was consistent with the previous study [27]. Compared with the CBA-140-1 sample, the CBA-120-1 sample exhibits a higher depth due to the 'small bending angle' inducing more deformation. For CBA-120-2 sample, the highest depth of coarse grains can be attributed to the more deformation induced by the 2 nd pass continuous bending process.
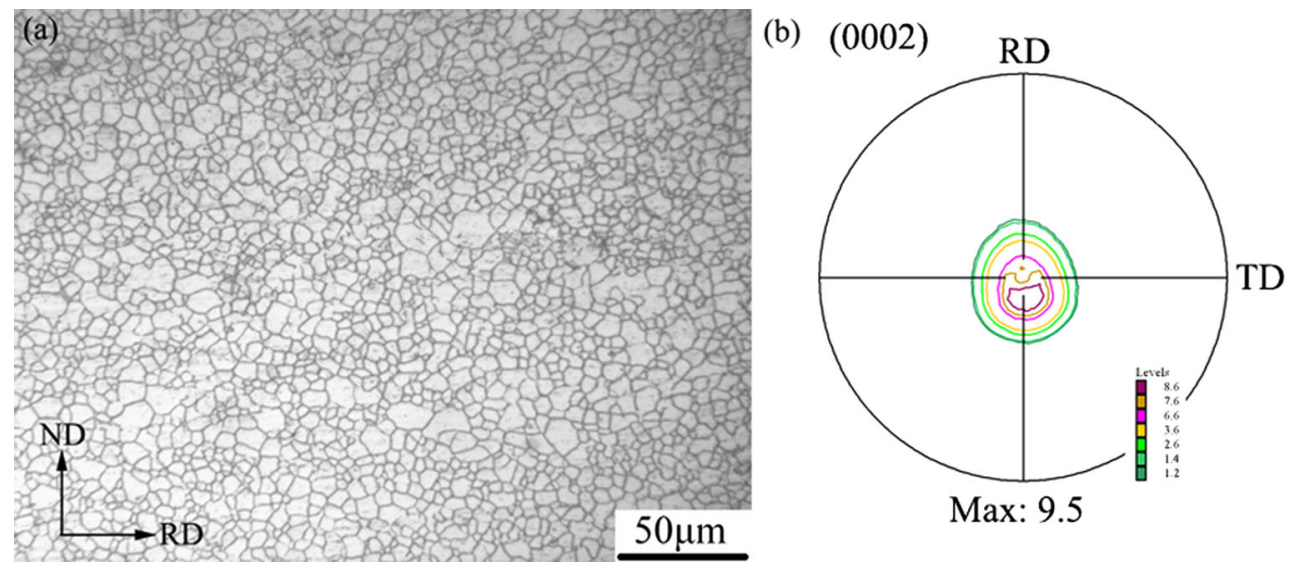

Fig. 2 a Optical microstructure, $\mathbf{b}(0002)$ pole figure of as-received sample 

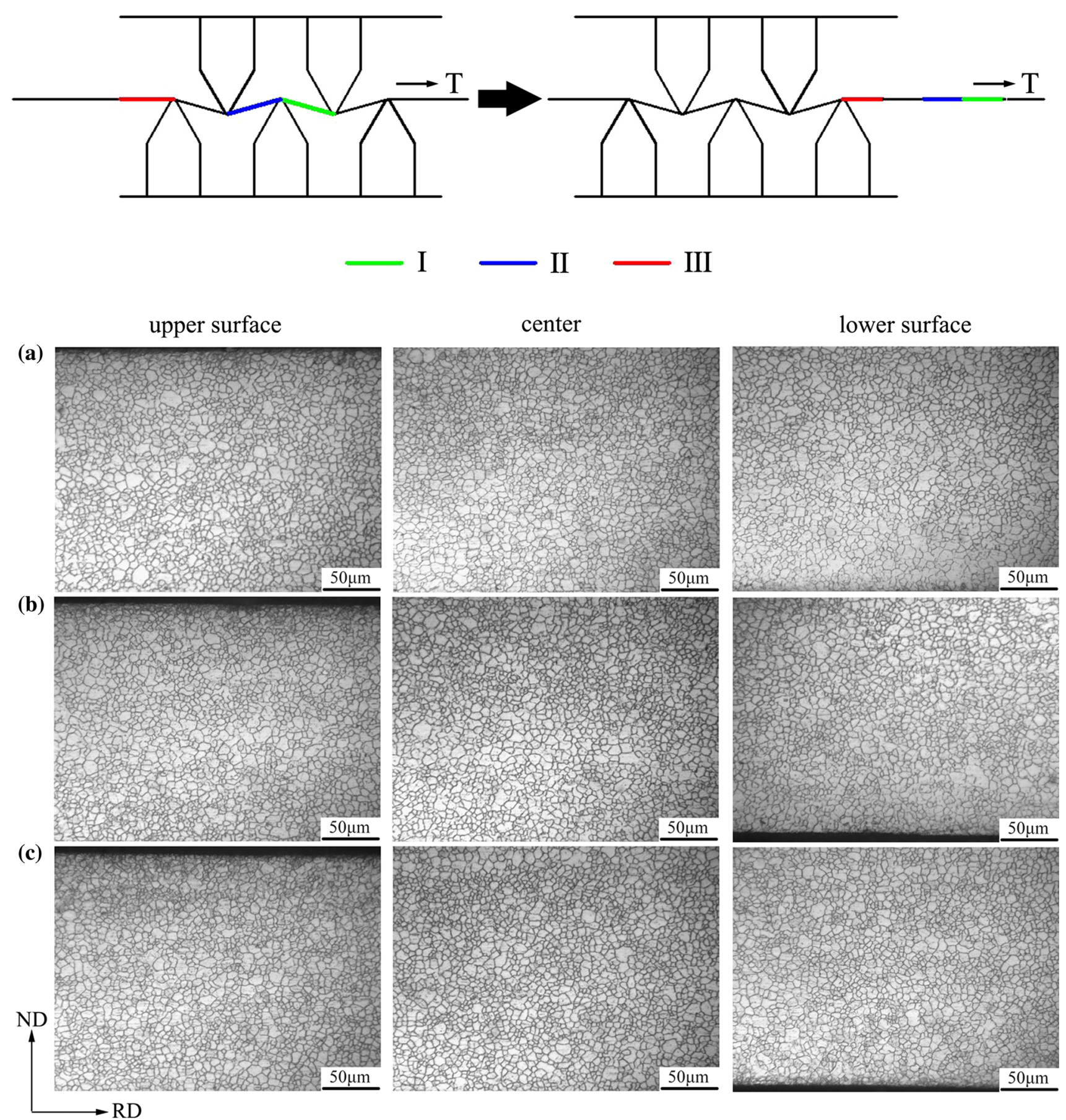

Fig. 3 Microstructures of sheets in different regions. a I region, b II region, c III region during CB-120-1 process

(0002) pole figures of CBA samples measured by XRD are shown in Fig. 5. Compared with the as-received sample as shown in Fig. 2b, the CBA samples exhibit a RD-tilt basal texture after $\mathrm{CB}$ process followed by annealing, indicating that $\mathrm{c}$-axes of more grains became inclined from ND toward RD. Due to the tilted basal poles, the dislocations with $\mathrm{a}<\mathrm{c}>$ Burgers vector component were needed during CB process. For the absence of twins, the $<c+a>$ pyramidal slip is needed and is also favorably oriented for the chosen deformation geometry [28]. The activation of $<\mathrm{c}+\mathrm{a}>$ slip leads to an increase tendency of basal poles rotate from ND toward RD. Kavyani et al. [25] indicated that pyramidal $<\mathrm{c}+\mathrm{a}>$ slip allowed $\mathrm{c}$-axis compression and the basal poles rotated away from ND toward RD. The similar results were obtained that the RD-tilt texture were obtained by the activity of pyramidal $<\mathrm{c}+\mathrm{a}>$ slip model during rolling [7, 23, 29-31]. Thus, the tilt of basal poles was most likely attributed to pyramidal $<\mathrm{a}+\mathrm{c}>$ slip. In 


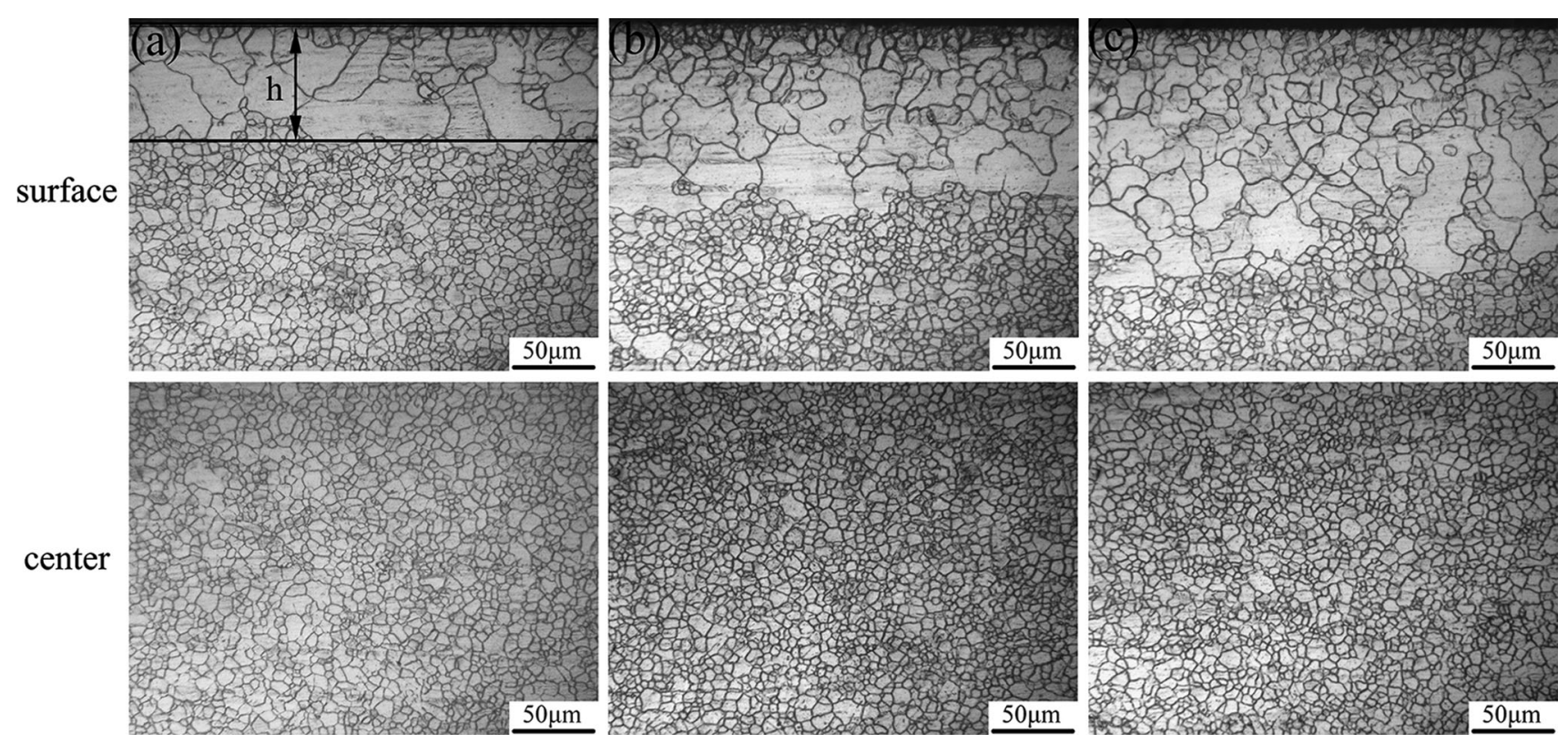

Fig. 4 Microstructures of a CBA-140-1, b CBA-120-1, c CBA-120-2 samples

(a)

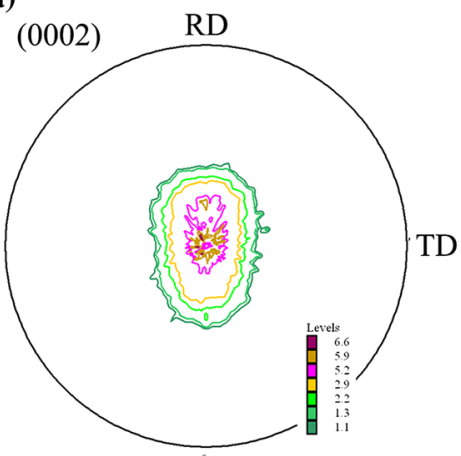

Max: 7.3 (b)

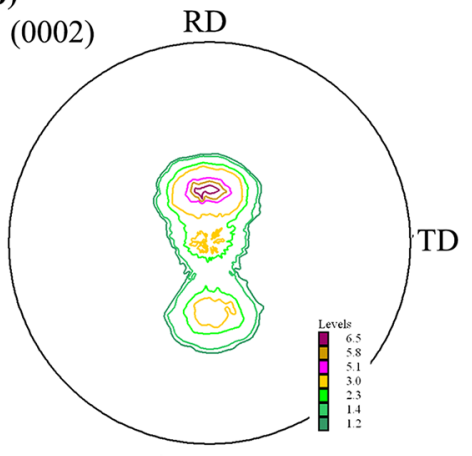

Max: 7.0 (c)

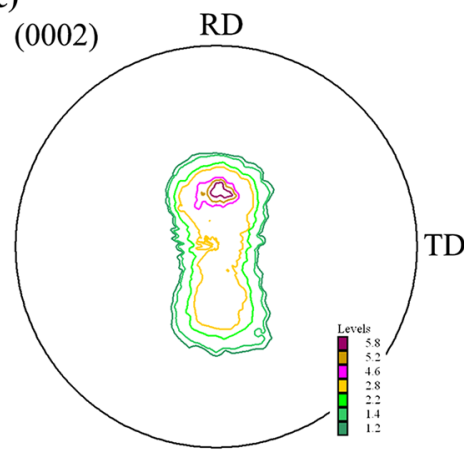

Max: 6.3

Fig. 5 The (0002) pole figures of a CBA-140-1, b CBA-120-1, c CBA-120-2 samples

addition, this obvious extra spreading of basal poles could also introduce an intensity decreasing in magnesium alloys. The maximum basal texture intensity was obviously weakened with the values of $7.3,7.0$ and 6.3 , respectively, indicating that the texture intensity decreased with the bending angle decreasing and repetitive passes increasing. Therefore, it is suggested that the CB process followed by annealing can be an effective approach to modify the basal texture.

\subsection{Mechanical Properties and Formability}

The typical tensile true stress-strain curves of the AS, CBA-140-1, CBA-120-1 and CBA-120-2 samples measured at room temperature are shown in Fig. 6. The tensile data such as yield strength (YS), ultimate tensile strength (UTS) and fracture elongation (FE) are summarized in
Table 1. Compared with the AS sample, the YS of CBA samples decreased from 183 to 140,127 and $112 \mathrm{MPa}$, respectively. The UTSs decreased slightly from 334 to 328 , 315 and $311 \mathrm{MPa}$, respectively. This indicated that the $\mathrm{CB}$ process had a strong effect on the YS but not on the UTS. The yield strength drop is most likely attributed to the two reasons: the RD-tilt and weakened basal texture and abnormal growth grains. Firstly, previous studies suggested that $<\mathrm{a}>$ basal slip has a great influence on the yield strength due to its lowest CRSS. A weak basal texture can result in a large Schmid factor of basal $<\mathrm{a}>$ slip due to the inclination of basal poles to RD and thus lead to the lower yield strengths of CBA samples [10, 32, 33]. Secondly, the coarse-grained microstructure near surface is another reason for the yield strength decreasing. According to Refs. $[22,34]$, the yield strength in tension followed the HallPetch relationship, $\sigma=\sigma_{0}+k d^{-1 / 2}$. It can be seen that the 


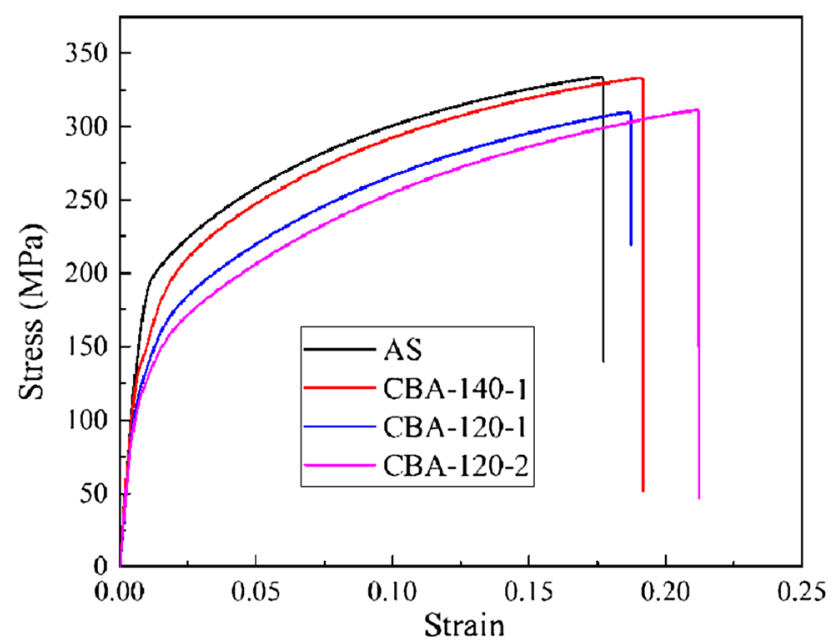

Fig. 6 True stress-strain curves of different samples

yield strength $\sigma$ depends on the average grain size $d$. This indicated that the abnormal grain growth near the surface region leads to the lower yield strength of CBA samples. The fracture elongations of CBA samples increased from 16.5 to $18.2,17.8$ and $19.2 \%$ compared to the AS sample, respectively. The enhancement of FE can be attributed to the weakened basal texture with a larger Schmid factor. For magnesium alloys, the smaller Schmid factor means that the activity of basal slip is more difficult leading to strain localization and premature shear failure [35]. In contrast, the thinning can be accommodated more easily by the higher activation of basal $<\mathrm{a}>$ slip during tension, leading to the high fracture elongation of CBA samples.

As shown in Fig. 7, we can see that CBA samples exhibit lower $r$-values of $2.13,1.83$ and 1.67, and larger $n$ values of $0.26,0.29$ and 0.30 than those of AS sample with the $r$-value of 2.98 and $n$-value of 0.23 . It is well known that texture generally affects the $r$-value of magnesium and its alloys. As $r=\omega_{\mathrm{w}} / \omega_{\mathrm{t}}$, where $\omega_{\mathrm{w}}$ and $\omega_{\mathrm{t}}$ represent the strain in width and thickness, respectively. For magnesium alloy sheets, the high $r$-value results from a large width strain or a small thickness strain. The magnesium alloy sheets with strong basal texture, basal slip is hardly activated during tensile deformation. Thus, the width strain is

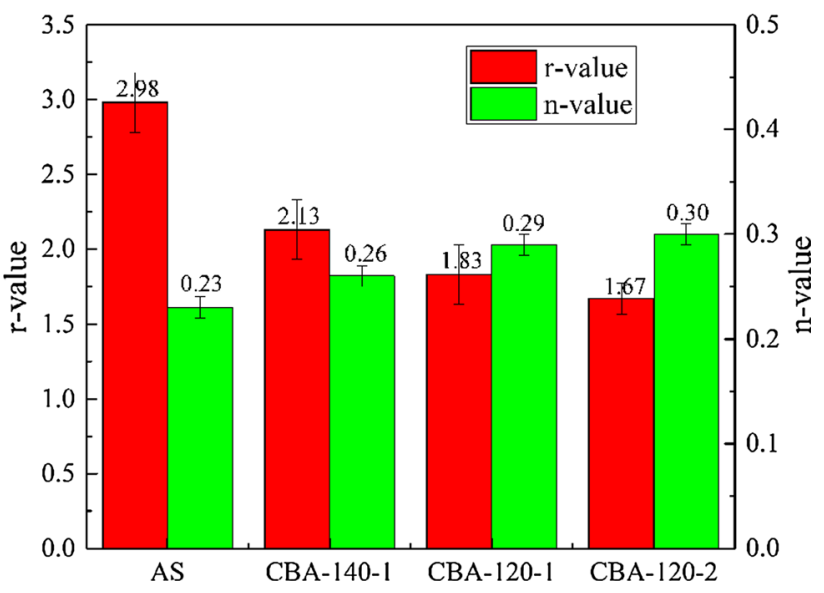

Fig. 7 Lankford values ( $r$-value) and strain hardening exponent values ( $n$-value) of various samples

dominated by prismatic slip and the thickness strain is dominated by pyramidal $<\mathrm{c}+\mathrm{a}>$ slip with the highest CRSS, resulting in a higher $r$-value. For the CBA samples with a RD-tilt basal texture, the thickness strain could be coordinated by basal $<\mathrm{a}>$ slip with the lowest CRSS, resulting the decrease of $r$-value. The similar tendency can be found in different speed rolling (DSR) process [10, 33]. In addition, the increase of $n$-value is originated from the weakened basal texture, since more grains would be favorably oriented for deformation. The large $n$-values lead to a low sensitivity to strain localization in the tension, and this in turn contributes to the increase in the fracture elongation.

Figure 8 shows the Erichsen values (IE) of different samples. Due to the strong basal texture, the IE of AS sample is only $2.3 \mathrm{~mm}$, which means poor formability at room temperature. The grains with strong texture in magnesium alloys stayed in a hard orientation to be deformed since the Schmid factor in the basal plane is near to zero. This results in stress localization and premature shear failure [35]. The sheets obtained by CB process followed by annealing exhibit superior stretch formability at room temperature. The IEs of CBA samples increased from 2.3 to $4.5,5.1$, and $5.4 \mathrm{~mm}$, increased by 96,122 and $135 \%$, respectively. The increase of IE can be attributed to the

Table 1 Mechanical properties of various samples

\begin{tabular}{|c|c|c|c|c|c|c|c|}
\hline \multirow[t]{2}{*}{ Samples } & \multicolumn{3}{|c|}{ YS (MPa) } & \multicolumn{3}{|c|}{ UTS (MPa) } & \multirow[t]{2}{*}{ FE $(\%)$} \\
\hline & $\mathrm{RD}$ & $45^{\circ}$ & TD & $\mathrm{RD}$ & $45^{\circ}$ & TD & \\
\hline AS & $183 \pm 2$ & $200 \pm 3$ & $216 \pm 1$ & $334 \pm 5$ & $323 \pm 7$ & $330 \pm 10$ & $16.5 \pm 0.5$ \\
\hline CBA-140-1 & $140 \pm 1$ & $165 \pm 2$ & $196 \pm 1$ & $328 \pm 5$ & $321 \pm 8$ & $336 \pm 7$ & $18.2 \pm 0.5$ \\
\hline CBA-120-1 & $127 \pm 2$ & $157 \pm 1$ & $191 \pm 3$ & $315 \pm 6$ & $303 \pm 5$ & $331 \pm 7$ & $17.8 \pm 0.5$ \\
\hline CBA-120-2 & $112 \pm 1$ & $151 \pm 1$ & $186 \pm 2$ & $311 \pm 4$ & $307 \pm 3$ & $313 \pm 6$ & $19.2 \pm 0.5$ \\
\hline
\end{tabular}




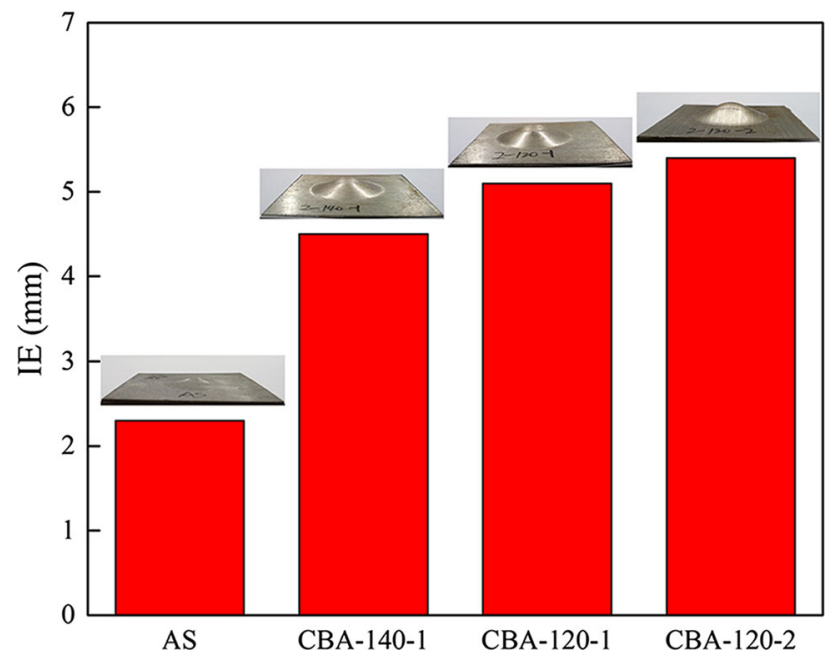

Fig. 8 Average values of IE of various samples

effects of $r$-value and $n$-value, which are related to the basal texture. The smaller $r$-values and larger $n$-values enhanced the capability to reduce the thickness of sheet during the stretch forming. Comparing with the uniaxial tension, the thickness strain is necessary for the form deformation under biaxial tensile stress [33]. The smaller $r$ value in weakened and tilted texture means that the more grains orientation is favorable for basal slip in thickness strain and that in turn enhances the formability of magnesium alloy sheets. $n$-value also plays an important role in controlling plastic instability in sheet forming. The larger $n$-value of the sheets is more enhanced local strain ability of the sheet during forming and increases the uniform elongation before fracture, resulting in the improvement of the formability. Additionally, not only the texture but also the grain size can affect the formability. The abnormal growth grains in the surface part may be in favor of improving the formability. The deform twinning enhanced by the abnormal grain growth can accommodate the strain of thickness [16].

The room temperature tensile fracture surfaces of the different samples are shown in Fig. 9. It is well known that magnesium alloys are inclined to form of cleavage fracture
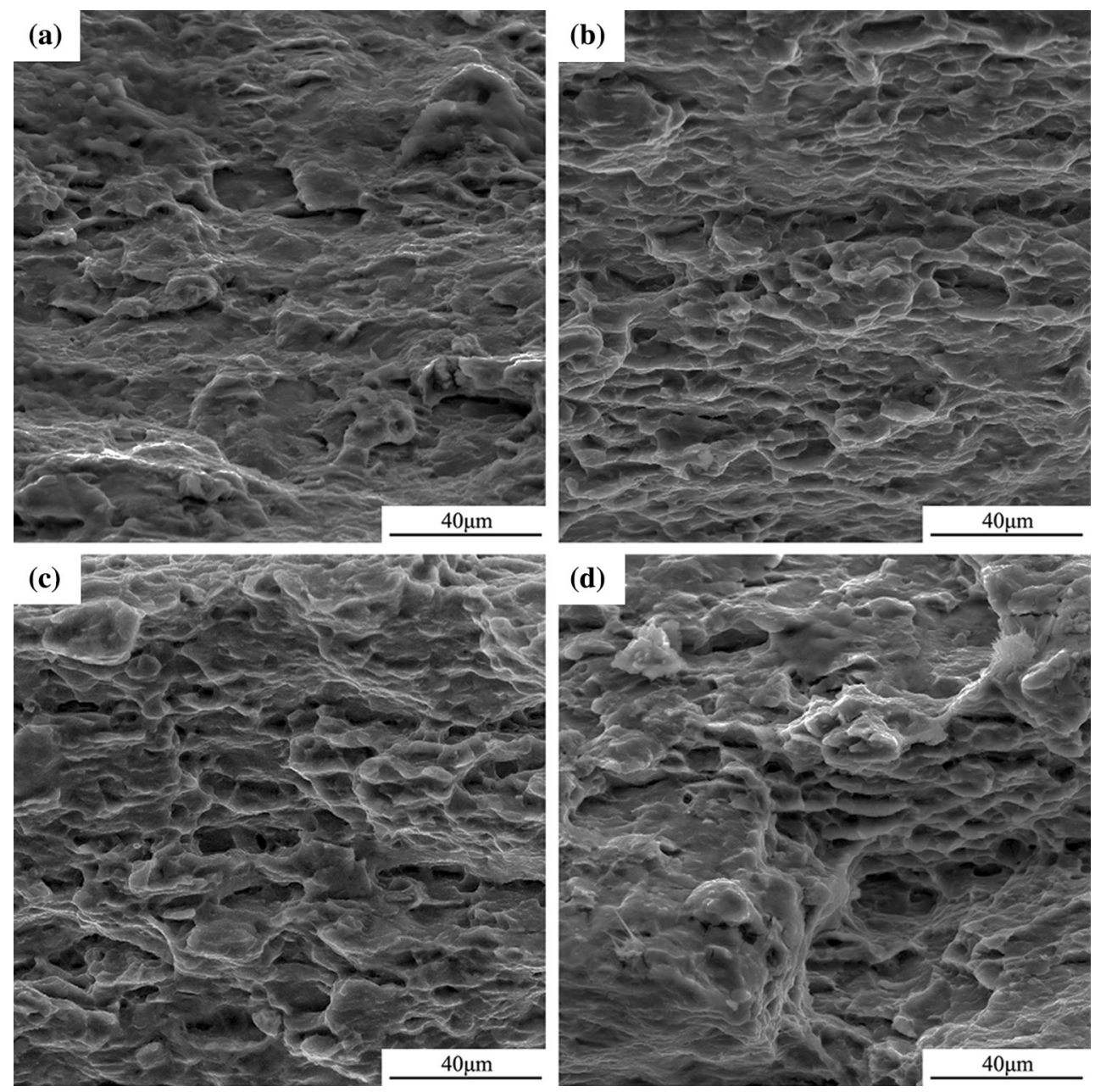

Fig. 9 SEM images of the tensile fracture surface of a AS, b CBA-140-1, c CBA-120-1, d CBA-120-2 samples 
or quasi-cleavage fracture due to their limited slip systems $[1,36,37]$. As can be seen in Fig. 9a, the fracture surface of AS sample showed a quasi-cleavage characteristic with a small amount of shallow dimples. This means that AS sample at room temperature exhibits a strong tendency of brittle fracture. While CBA samples showed numerous dimples and some tearing edges, CBA-120-1 and CBA120-2 samples exhibited a few of deep dimples which belong to the ductile failure [38]. The different fracture features of samples, after tension to fracture, should be associated with the difference in their deformation mechanisms. For AS sample with a strong basal texture, the prismatic slip dominated the tensile deformation. The CBA samples with the weakened and RD-tilt basal texture; however, the basal slip can be easily activated during tension. Due to the lower CRSS of basal slip than that of prismatic slip, CBA samples exhibited more tendency of ductile failure. Therefore, it is supposed that accumulation of plastic deformation in magnesium alloy sheets is promoted by continuous bending process followed by annealing.

\section{Conclusions}

Microstructure and mechanical properties of AZ31 magnesium alloy sheets underwent continuous bending process and annealing have been investigated in the present work. The major conclusions can be drowning as following:

1. The microstructure of all samples underwent different bending times exhibited no existence of twins. After continuous bending and annealing, the grains abnormally grew in the surface part and the grains in the center changed not much.

2. All the CBA samples exhibited a RD-tilt basal texture, and the texture intensity decreased with the bending angle decreasing and the repetitive passes increasing.

3. Due to the weakened basal texture, the CBA samples exhibited a decrease in YS and an increase in FE. The CBA-120-2 sample had the lowest YS of $112 \mathrm{MPa}$ and highest fracture elongation of $19.2 \%$. Compared with the AS sample, the CBA samples possess a smaller $r$ value and a larger $n$-value.

4. The highest of IE was $5.4 \mathrm{~mm}$, greatly increased by $\sim 135 \%$, which was attributed to lower yield strength, larger fracture elongation, a smaller $r$-value and a larger $n$-value related to the weakened basal texture. It is suggested that the continuous bending process followed by annealing is a short time and effective method for enhance ductility of magnesium alloy sheets.
Acknowledgements This work was financially supported by the National Natural Science Foundation of China (Nos. 51671041 and 51531002); the National Key Research and Development Plan (No. 2016YFB0301104); Demonstrative Project of Chongqing Science and Technology Commission (No. CSCT2014FAZKTJCSF50004); and Fundamental Research Funds for the Central Universities (No. 106112017CDJPT280001).

\section{References}

[1] L. Zheng, H. Nie, W. Liang, H. Wang, Y. Wang, J. Magnes. Alloys 4, 115-122 (2016)

[2] I. Aatthisugan, A.R. Rose, D.S. Jebadurai, J. Magnes. Alloys 5(1), 20-25 (2017)

[3] H. Zhang, Y. Yan, J. Fan, W. Cheng, H.J. Roven, B. Xu, H. Dong, Mater. Sci. Eng. A 618, 540-545 (2014)

[4] J. Koike, T. Kobayashi, T. Mukai, H. Watanabe, M. Suzuki, K. Maruyama, K. Higashi, Acta Mater. 51, 2055-2065 (2003)

[5] M. Mabuchi, Y. Chino, H. Iwasaki, Mater. Trans. 44, 490-495 (2003)

[6] H. Zhang, G. Huang, J. Fan, H.J. Roven, B. Xu, H. Dong, J. Alloys Compd. 615, 302-310 (2014)

[7] P. Liu, H. Jiang, Z. Cai, Q. Kang, Y. Zhang, J. Magnes. Alloys 4, 188-196 (2016)

[8] H. Zhang, Y. Liu, J. Fan, H.J. Roven, W. Cheng, B. Xu, H. Dong, J. Alloys Compd. 615, 687-692 (2014)

[9] X. Huang, K. Suzuki, A. Watazu, I. Shigematsu, N. Saito, J. Alloys Compd. 479, 726-731 (2009)

[10] X. Huang, K. Suzuki, A. Watazu, I. Shigematsu, N. Saito, J. Alloys Compd. 470, 263-268 (2009)

[11] Y. Chino, K. Sassa, A. Kamiya, M. Mabuchi, Mater. Sci. Eng. A 473, 195-200 (2008)

[12] H. Zhang, G. Huang, L. Wang, H.J. Roven, F. Pan, J. Alloys Compd. 575, 408-413 (2013)

[13] J. Suh, J. Victoria-Hernandez, D. Letzig, R. Golle, S. Yi, J. Bohlen, W. Volk, J. Mater. Proc. Technol. 217, 286-293 (2015)

[14] J. Suh, J. Victoria-Hernández, D. Letzig, R. Golle, W. Volk, Mater. Sci. Eng. A 669, 159-170 (2016)

[15] B. Song, G. Huang, H. Li, L. Zhang, G. Huang, F. Pan, J. Alloys Compd. 489, 475-481 (2010)

[16] H. Zhang, G.-S. Huang, B. Song, L. Zhang, D.-Q. Kong, Trans. Nonferrous Metal. Soc. 21, 844-850 (2011)

[17] T.Z. Han, G.S. Huang, Y.G. Wang, G.-G. Wang, Y.-C. Zhao, F.S. Pan, Trans. Nonferrous Metal. Soc. 26, 2043-2050 (2016)

[18] T. Han, G. Huang, Y. Wang, G. Wang, Y. Zhao, F. Pan, Prog. Nat. Sci. Mater. Int. 26, 97-102 (2016)

[19] G. Huang, L. Wang, H. Zhang, Y. Wang, Z. Shi, F. Pan, Mater. Lett. 98, 47-50 (2013)

[20] L. Wang, G. Huang, H. Zhang, Y. Wang, L. Yin, J. Mater. Proc. Technol. 213, 844-850 (2013)

[21] M.R. Barnett, Scr. Mater. 59, 696-698 (2008)

[22] M. Barnett, Z. Keshavarz, A. Beer, D. Atwell, Acta Mater. 52, 5093-5103 (2004)

[23] M. Knezevic, A. Levinson, R. Harris, R.K. Mishra, R.D. Doherty, S.R. Kalidindi, Acta Mater. 58, 6230-6242 (2010)

[24] T. Al-Samman, G. Gottstein, Mater. Sci. Eng. A 490, 411-420 (2008)

[25] M. Kavyani, G.R. Ebrahimi, M. Sanjari, M. Haghshenas, J. Magnes. Alloys 4, 89-98 (2016)

[26] Y. Takayama, J.A. Szpunar, H.-T. Jeong, Mater. Trans. 42, 2050-2058 (2001)

[27] G.-S. Huang, H. Zhang, X.-Y. Gao, B. Song, L. Zhang, Trans. Nonferrous Metal. Soc. 21, 836-843 (2011) 
[28] Z.-Y. Liu, T.-T. Huang, W.-J. Liu, S. Kang, Trans. Nonferrous Metal. Soc. 26, 378-389 (2016)

[29] F. Guo, D. Zhang, X. Yang, L. Jiang, S. Chai, F. Pan, Mater. Sci. Eng. A 607, 383-389 (2014)

[30] W.P. Jia, X.D. Hu, H.Y. Zhao, D.Y. Ju, D.L. Chen, J. Alloys Compd. 645, 70-77 (2015)

[31] Y. Zou, L. Zhang, H. Wang, X. Tong, M. Zhang, Z. Zhang, J. Alloys Compd. 669, 72-78 (2016)

[32] T. Zhou, Z. Yang, D. Hu, T. Feng, M. Yang, X. Zhai, J. Alloys Compd. 650, 436-443 (2015)

[33] H. Zhang, G. Huang, L. Wang, H.J. Roven, Z. Xu, F. Pan, Scr. Mater. 69, 49-52 (2013)
[34] G. Mann, J. Griffiths, C. Cáceres, J. Alloys Compd. 378, 188-191 (2004)

[35] S.R. Agnew, Ö. Duygulu, Int. J. Plast. 21, 1161-1193 (2005)

[36] Z. Li, J. Dong, X.Q. Zeng, C. Lu, W.J. Ding, Mater. Sci. Eng. A 466, 134-139 (2007)

[37] F. Lu, A. Ma, J. Jiang, J. Chen, D. Song, Y. Yuan, J. Chen, D. Yang, J. Alloys Compd. 643, 28-33 (2015)

[38] Q. Yang, B. Jiang, X. Li, H. Dong, W. Liu, F. Pan, J. Magnes. Alloys 2, 8-12 (2014) 